\title{
Effects of Precursors on Brain Neurotransmitter Synthesis and Brain Functions
}

\author{
J. D. Fernstrom \\ Department of Nutrition and Food Science, Massachusetts Institute of Technology, Cambridge, Massachusetts, USA
}

\begin{abstract}
This paper reviews factors which influence the levels of aminergic transmitters in the brain. In particular precursor availability to the brain influences the rates of synthesis of serotonin, the catecholamines, and acetylcholine by brain neurons. The diet readily influences brain neurotransmitter formation via this mechanism. At present, the importance of this relationship to body regulation is not well understood. Nonetheless, precursors (tryptophan, tyrosine, choline, and lecithin) have begun to find uses as therapeutic agents in the treatment of disease states involving diminished transmitter formation and release. Hopefully, these compounds will find a wide range of uses, as they lack many of the side effects that accompany the use of drugs.
\end{abstract}

Key words: Tryptophan, tyrosine, choline, serotonin, catecholamines, acetylcholine, diabetes, blood pressure, pituitary hormones, neurologic disorders

The rates of synthesis of several brain neurotransmitters depend in part on the availability to neurons of the appropriate precursor molecules. Notably, the administration of tryptophan, tyrosine, or choline stimulates, under appropriate conditions, the formation of serotonin, the catecholamines, or acetylcholine, respectively. As a consequence, such treatments alter brain and body functions influenced by neurons utilizing these transmitters. In this article, the evidence supporting these precursor-product relationships will be reviewed, and several of the physiological-behavioral consequences will be discussed.

\section{Serotonin}

Serotonin (5-hydroxytryptamine; 5HT) is synthesized from the essential amino acid L-tryptophan, by the two-step pathway outlined in Figure 1 (top panel). The first reaction, tryptophan hydroxylation, is rate-limiting [1]. Moreover, the rate of this reaction varies directly with brain tryptophan levels because the hydroxylase enzyme is not fully saturated with substrate at normal brain tryptophan concentrations [2]. Hence, acute (or chronic) increases in brain tryptophan elevate brain 5HT (and the levels of its principal metabolite, 5-hydroxyindoleacetic acid, 5HIAA), and decreases in brain tryptophan reduce brain 5HT and 5HIAA $[3,4]$.

Because changes in brain tryptophan are soon reflected as alterations in serotonin synthesis, factors that influence tryptophan uptake into brain should be potentially important determinants of brain $5 \mathrm{HT}$ formation. In normal animals, the single most important factor influencing tryptophan access to brain is the large neutral amino acid transport (LNAA) system, located at the blood-brain barrier [5]. Tryptophan is taken up into brain by this carrier system, as are other large neutral amino acids such as tyrosine, phenylalanine, and the branched-chain amino acids. The transport system is competitive [5]; as a consequence, tryptophan uptake into brain (and serotonin formation) depends not simply on blood tryptophan levels, but also on the blood levels of these other LNAA competitors. Brain tryptophan can be raised either by raising blood tryptophan, or by lowering blood levels of the other LNAA; it can be lowered either by reducing blood tryptophan, or by raising blood LNAA levels [6].

While pharmacologic injections of tryptophan or other LNAA alter brain tryptophan (and 5HT), similar effects also occur physiologically through the act 
of eating [6]. The ingestion (by fasting animals) of a carbohydrate meal rapidly elevates brain tryptophan and serotonin; it does so by raising blood tryptophan, and lowering the blood levels of its competitors, the other LNAAs. Tryptophan thereby presumably gains a competitive advantage for brain uptake, and brain tryptophan and 5-hydroxyindole levels rise. These effects all depend on the secretion of insulin, which produces the changes in blood neutral amino acid pattern favorable to enhanced tryptophan uptake into brain. The competitive transport mechanism also explains why the ingestion of a protein containing meal usually does not elicit a rise in brain tryptophan. When protein is ingested, not only does blood tryptophan rise, but so do the levels of the other LNAA (unlike after the ingestion of carbohydrate alone). In fact, the relative increases in blood levels of tryptophan and the other LNAA are approximately equal. Hence, the competition for uptake at brain transport sites is unchanged, and brain tryptophan and 5-hydroxyindole levels are unaltered [7]. A variety of studies attest to the importance of the competitive uptake system in determining brain tryptophan and 5HT after the ingestion of any particular meal [8].

Although the protein and carbohydrate contents of a meal thus seem to be important factors in determining post-prandial brain tryptophan levels, fat content is not. The ingestion of moderate to large amounts of fat in a meal causes serum levels of the nonesterified fatty acids (NEFA) to rise, leading to competitive displacement of tryptophan molecules from their weak binding sites on serum albumin [9]. Although some data have been provided suggesting such displacement of tryptophan from albumin to cause robust increments in brain tryptophan [10], no such increases are seen when animals consume a high fat, protein-containing meal (which causes a large rise in serum-free tryptophan) [9]. This result is not surprising in retrospect. The affinity and capacity of the brain transport carrier for tryptophan exceed those of albumin; almost all tryptophan molecules (bound and free) are thus readily accessible to the transport carrier as they pass through brain capillaries [11]. Hence, the major determinant of tryptophan uptake is competition among the LNAA and tryptophan, not binding of tryptophan to serum albumin. (Under certain pharmacologic conditions, where the bulk of the tryptophan molecules bound to albumin are displaced, brain tryptophan may be increased [10]. However, such pharmacologic curiosities do not predict normal physiology.)

If competition among the LNAA for brain uptake is important physiologically in determining brain tryptophan levels, it also figures predominantly in certain pathologic conditions. For example, brain tryptophan and serotonin are reduced in experimental phenylketonuria (PKU). These changes reflect the high circulating levels of phenylalanine, which undoubtedly diminish tryptophan uptake into brain [5]. Another disease state to receive recent attention is diabetes. In diabetes, blood levels of the branchedchain amino acids are very high [12]. Such abnormal elevations in the blood levels of these amino acids should and do diminish tryptophan uptake into brain, and brain tryptophan levels [13, 14]. In addition, because of the absence of insulin secretion, blood LNAA levels, and therefore brain indoles, do not change normally after carbohydrate ingestion. For example, Table 1 shows that glucose intubation of fasting normal rats elevated the blood ratio of tryptophan to the sum of its LNAA competitors (this ratio is a simple predictor of the state of the competition for brain uptake between tryptophan and the
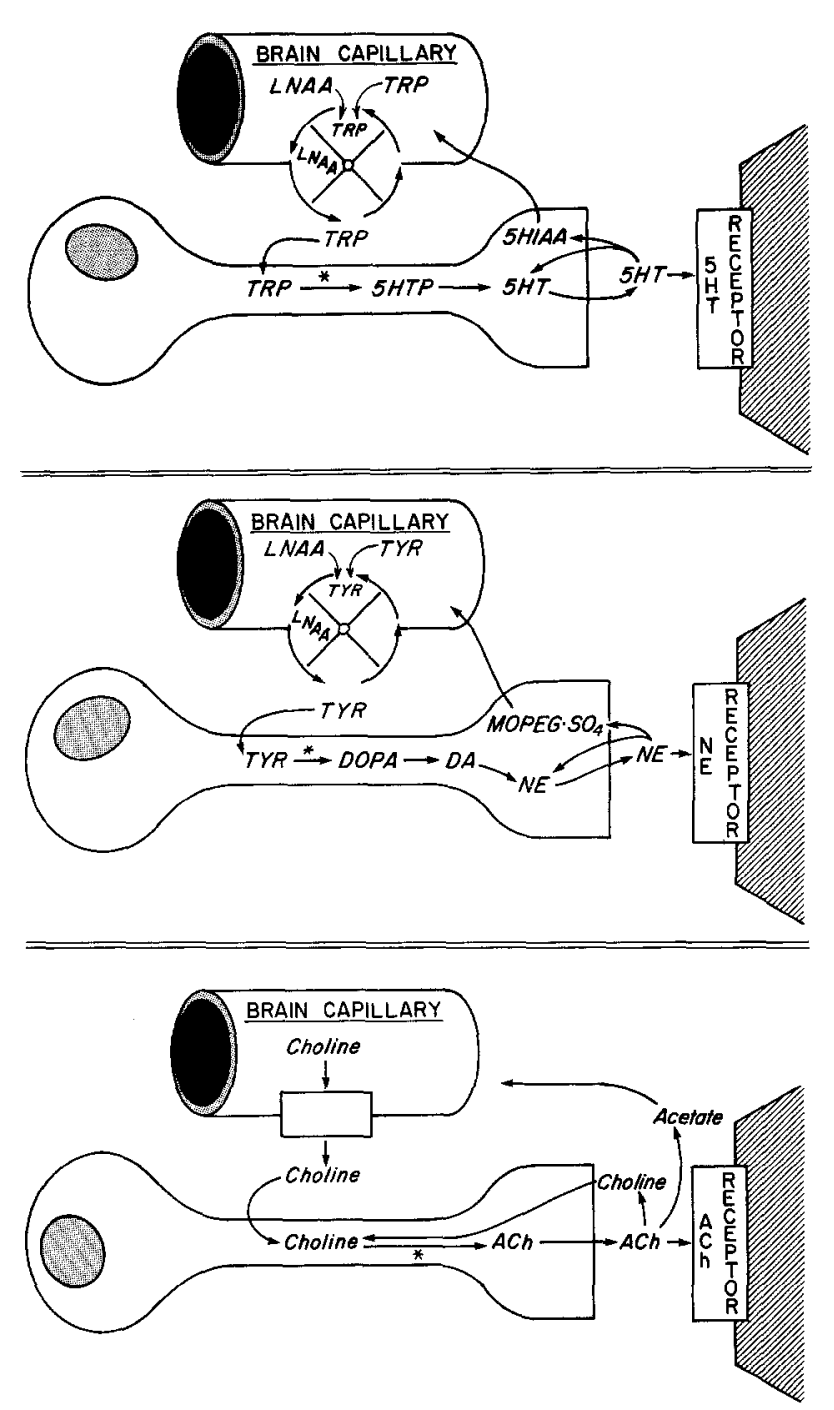
other LNAA in blood), and brain tryptophan and 5hydroxyindoles [15]. In diabetic rats, no increments in the serum tryptophan ratio, brain tryptophan, or brain 5-hydroxyindoles occurred following glucose intubation. That insulin was central to such changes in the diabetic rats was strengthened by the observa-

Table 1. Effects of glucose intubation of fasting normal and diabetic rats on brain indole levels

\begin{tabular}{lll}
\hline Indole & Fasting & Glucose \\
\hline Serum tryptophan ratio & & \\
$\quad$ Normal rats & $0.16 \pm 0.01$ & $0.30 \pm 0.02^{\mathrm{a}}$ \\
$\quad$ Diabetic rats & $0.09 \pm 0.01^{\mathrm{b}}$ & $0.13 \pm 0.04^{\mathrm{b}}$ \\
Brain tryptophan $(\mathrm{nmol} / \mathrm{g})$ & & \\
$\quad$ Normal rats & $26.50 \pm 2.00$ & $36.30 \pm 0.50^{\mathrm{a}}$ \\
$\quad$ Diabetic rats & $13.70 \pm 1.50^{\mathrm{b}}$ & $15.70 \pm 2.00^{\mathrm{b}}$ \\
Brain serotonin $(\mathrm{nmol} / \mathrm{g})$ & & \\
$\quad$ Normal rats & $4.11 \pm 0.07$ & $4.48 \pm 0.05^{\mathrm{a}}$ \\
$\quad$ Diabetic rats & $4.16 \pm 0.18$ & $4.30 \pm 0.07$ \\
Brain 5-HIAA $(\mathrm{nmol} / \mathrm{g})$ & & \\
$\quad$ Normal rats & $3.47 \pm 0.14$ & $4.38 \pm 0.18^{\mathrm{a}}$ \\
$\quad$ Diabetic rats & $2.95 \pm 0.13^{\mathrm{b}}$ & $3.25 \pm 0.15^{\mathrm{b}}$ \\
\end{tabular}

Normal and diabetic rats were fasted overnight, and the following morning were intubated with a $2 \mathrm{M}$ glucose solution $(1 \mathrm{ml} / 100 \mathrm{~g})$. They were killed $2 \mathrm{~h}$ later. Control animals continued to fast during the $2 \mathrm{~h}$ period. Data are presented as the means \pm standard errors

${ }^{a} \mathrm{P}<0.05$ that value differs significantly from corresponding fasting value;

b $\mathrm{P}<0.05$ that value differs significantly from corresponding value in normal rats (adapted from [15])

Fig. 1. Precursor dependence of neurotransmitter synthesis in the mammalian brain. Top Panel: Relationship of tryptophan availability to serotonin (5-HT) synthesis. Tryptophan (TRP) levels in brain directly influence the rate at which the amino acid is converted to 5-HT. The uptake of tryptophan into brain is via a competitive transport mechanism shared with other large neutral amino acids (LNAA). See text for details. 5-HTP $=5$-hydroxytryptophan; 5-HIAA $=5$-hydroxyindoleacetic acid; * indicates ratelimiting step in 5-HT formation, tryptophan hydroxylation. Middle Panel: Relationship of tyrosine (TYR) availability to norepinephrine (NE) synthesis. TYR levels in brain influence directly the rate at which the amino acid is converted to NE. The uptake of TYR into brain is, as for TRP, via a competitive uptake mechanism shared with other LNAA. See text for details. DOPA = dihydroxyphenylalanine; $\mathrm{DA}=$ dopamine; $\mathrm{MOPEG}-\mathrm{SO}_{4}=\mathrm{me}-$ thoxyhydroxyphenethylglycol-sulfate, a principal NE metabolite in brain; * indicates rate-limiting step in NE formation, tyrosine hydroxylation. Bottom Panel: Relationship of choline availability to acetylcholine (ACh) synthesis. Choline levels in brain neurons influence directly the rate at which the choline is converted to $\mathrm{ACh}$. The uptake of choline into brain is via a mechanism that appears to be specific for choline. Choline in blood derives principally from two sources, choline synthesized in the liver, and choline liberated during digestion and absorption of dietary lecithin * indicates rate-limiting step in ACh formation, choline acetylation. See text for details tion that the administration of insulin to diabetic rats ingesting carbohydrates was associated with increases in the serum tryptophan ratio, brain tryptophan, and brain 5-hydroxyindoles. Hence, the diabetic brain, because of the lack of insulin secretion by the pancreas, is deprived of a change in composition that occurs each time a normal animal consumes carbohydrate. Perhaps this alteration in diabetes ultimately contributes to the observed changes in brain functions associated with this disease.

What purpose might be served in the body's economy by having the formation of a brain neurotransmitter tied to variations in blood LNAA levels, especially when blood LNAA levels can be altered by so common an activity as eating? At present, this question is answered mainly by speculation: perhaps, by this mechanism, the brain receives information about peripheral metabolic state, or composition of the diet (i. e., the rise in brain serotonin level, and thus presumably release, may be a signal to the brain that carbohydrates have been consumed $[6,16]$ ). Data are available suggesting that serotonin neurons participate in the regulation of food intake, and that diet-induced changes in brain serotonin can influence directly protein selection in the diet by animals over a several week period [17]. However, much further work is needed to establish the robustness of such a relationship.

On the other hand, the evidence is becoming very convincing that pharmacologic alterations of brain tryptophan levels, and thus of brain 5HT levels, influence brain functions controlled in part by serotonergic neurons. This type of finding implies that when the diet does alter brain serotonin by influencing brain tryptophan, it probably also alters serotonin neurotransmission. Examples of the effects of tryptophan administration include alterations in the sleep of rats and humans [18], in blood pressure in rats [19], and in blood growth hormone levels in both rats [20] and man [21]. An example of the effect of tryptophan on serum growth hormone is shown in Table 2. Injection of the amino acid at 0800 led to approximately a two-fold enhancement of blood GH levels over the succeeding 10 -hour period. This effect could be blunted by co-administration of valine (another LNAA), which blocked tryptophan uptake into brain following its injection. These data are consistent with other pharmacologic evidence showing serotonin neurons to facilitate GH secretion [22]. Thus, as also indicated in Table 2, the injection of a $5 \mathrm{HT}$ receptor antagonist reduces serum $\mathrm{GH}$ levels. Moreover, 5HT agonists have been shown to raise blood $\mathrm{GH}$, and the administration of $\mathrm{p}$-chlorophenylalanine, an inhibitor of serotonin synthesis, depresses blood GH levels $[23,24]$. 
Table 2. Effect of tryptophan or metergoline on plasma growth hormone levels in the rat

\begin{tabular}{lc}
\hline Treatment & $\begin{array}{l}\text { Mean plasma } \\
\text { GH levels }\end{array}$ \\
\hline & $(\mathrm{ng} / \mathrm{ml})$ \\
Saline & $77.4 \pm 9.3$ \\
Tryptophan $(100 \mathrm{mg} / \mathrm{kg})$ & $143.3 \pm 13.1^{\mathrm{a}}$ \\
Metergoline $(2 \mathrm{mg} / \mathrm{kg})$ & $44.1 \pm 8.7^{\mathrm{a}}$ \\
\hline
\end{tabular}

Groups of 5 male rats received saline, tryptophan, or metergoline at $0800 \mathrm{~h}$, following which blood samples were drawn at 15 minute intervals throughout the day until $1800 \mathrm{~h}$

Data are presented as the means \pm s.e.m., of the integrated values of plasma $\mathrm{GH}$ during the $0800-1800$ period

a $\mathrm{P}<0.01$ compared to saline values (from [42])

Thus, in summary, diet does influence brain serotonin formation, by modifying tryptophan uptake into brain, and brain tryptophan levels. Tryptophan-induced increases in brain serotonin do apparently increase $5 \mathrm{HT}$ release, as selected brain outputs under the influence of 5HT neurons are predictably altered by this treatment. The ultimate importance of these relationships awaits further clarification, although some data indicate food-induced changes in brain serotonin may function to regulate dietary selection of protein and/or carbohydrates.

\section{Norepinephrine and Dopamine}

The catecholamine neurotransmitters, dopamine and norepinephrine, are synthesized from tyrosine. The pathway outlined in Figure 1 (middle panel) is for neurons that utilize norepinephrine (NE); for dopamine neurons, the only differences are in the absence from neurons of dopamine-beta-hydroxylase, the enzyme which catalyzes the conversion of dopamine (DA) to NE, and in the metabolites produced from the transmitter. In noradrenergic neurons, methoxyhydroxyphenethylene-glycol-sulfate (MOPEG-SO ${ }_{4}$ ) is a major metabolite, while in dopaminergic neurons, homovanillic acid (HVA) and dihydroxyphenylacetic acid (DOPAC) are the major metabolites [25].

In both types of catecholamine neurons, the initial step in transmitter synthesis, the hydroxylation of tyrosine to dopa, is rate-limiting. A sizeable body of pharmacologic data suggests that the rate of tyrosine hydroxylation is controlled by end-product inhibition [25]. However, this is not the only mechanism that influences catecholamine synthesis. The hydroxylation rate is also influenced by precursor levels in brain, since tyrosine hydroxylase, like tryptophan hydroxylase, is not fully saturated with substrate at normal brain tyrosine concentrations [26]. It is thus possible, for example, to accelerate tyrosine hydroxylation in vivo by injecting tyrosine, and to reduce it by injecting another LNAA, like valine, which lowers brain tyrosine levels. (This latter effect follows from the fact that tyrosine is also an LNAA, and thus subject to the same competitive uptake system for brain as that described above for tryptophan.)

It has also been possible to demonstrate that, under certain circumstances, changes in brain tyrosine levels also affect the overall rates of DA and NE synthesis, as evidenced by the ability of tyrosine injection rapidly to raise brain MOPEG-SO $\mathrm{SO}_{4}$ levels [27], while not changing brain NE, and to increase regional brain DOPAC and HVA levels, while not altering dopamine concentrations [28]. For NE (DA has not yet been carefully studied), the ingestion of single meals also rapidly alters brain MOPEG-SO ${ }_{4}$ accumulation [29]. The particular effect of a meal on brain MOPEG-SO ${ }_{4}$ depends on how its ingestion modifies the blood levels of tyrosine relative to those of the LNAA competitors, and thus brain tyrosine levels [30]. It is again remarkable to find that the synthesis of yet another brain transmitter is readily influenced by precursor availability, as modulated by the diet. The potential physiological significance of this relationship is only now beginning to be explored.

Setting aside dietary effects, the administration of L-tyrosine alone produces a number of interesting effects in mammals. For example, its administration to spontaneously-hypertensive (SHR) rats elicits a sizeable drop in blood pressure (Fig. 2), an effect that is blunted by the co-administration of valine [27]. The tyrosine-induced fall in blood pressure is accompanied by an increase in brain MOPEG-SO ${ }_{4}$ level, largely confined to the brainstem. This result suggests that the antihypertensive effect of tyrosine is related to enhanced NE synthesis and release, and thus fits with other observations suggesting that the stimulation of central noradrenergic receptors reduces blood pressure [31].

Tyrosine administration can also lower serum prolactin levels under appropriate circumstances by a mechanism most likely involving dopaminergic neurons. Blood prolactin levels are elevated in animals treated chronically with reserpine (Table 3 ). The administration to such animals of tyrosine leads to a sizeable reduction in serum prolactin. This effect is accompanied by increases in DOPAC and HVA levels in several brain regions, including the hypothalamus (DA levels themselves do not change), suggesting increased DA synthesis and release [28]. These results are all consistent with other published data suggesting dopamine to function as a physiologic 


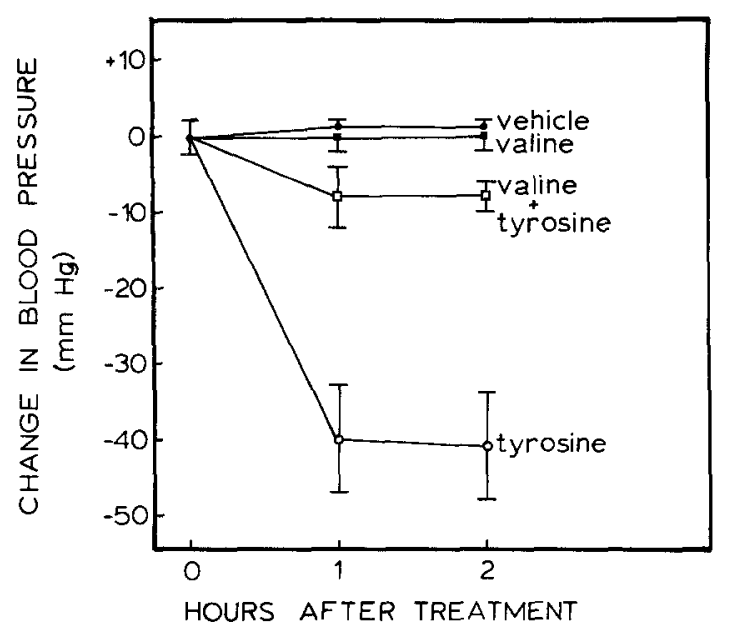

Fig. 2. Effect of tyrosine and valine on blood pressure in hypertensive rats. Tyrosine $(100 \mathrm{mg} / \mathrm{kg} ; 0.55 \mathrm{mmol} / \mathrm{kg})$ [0], valine $(0.55 \mathrm{mmol} / \mathrm{kg})[\mathbf{\square}]$, tyrosine plus valine $(0.55 \mathrm{mmol} / \mathrm{kg}$ each $)[\square]$, or vehicle [-] was administered to groups of four spontaneouslyhypertensive rats. Blood pressures were measured just before treatment and then at one-hour intervals afterwards. Data are expressed as the changes in blood pressure from baseline values (mean $\pm \mathrm{SEM}$ ). Tyrosine injection significantly reduced blood pressure $(\mathrm{P}<0.01)$; coadministration of valine with tyrosine blunted sizeably the tyrosine-induced fall in pressure. Valine injection alone was without effect on blood pressure. (From [27])

Table 3. Effect of tyrosine injection on serum levels of prolactin and hypothalamic levels of dopamine metabolites in reserpinized rats

\begin{tabular}{lccl}
\hline Treatment & $\begin{array}{l}\text { Serum } \\
\text { prolactin }\end{array}$ & $\begin{array}{l}\text { Hypo- } \\
\text { thalamic } \\
\text { DOPAC }\end{array}$ & $\begin{array}{l}\text { Hypo- } \\
\text { thalamic } \\
\text { HVA }\end{array}$ \\
\hline $\begin{array}{l}\text { No reserpine } \\
\text { Vehicle } \\
\text { Tyrosine }\end{array}$ & $52 \pm 13$ & $56 \pm 18$ & $187 \pm 20$ \\
$\begin{array}{l}\text { Chronic reserpine } \\
\text { Vehicle }\end{array}$ & $49 \pm 9$ & $85 \pm 21$ & $243 \pm 35$ \\
Tyrosine & $156 \pm 14$ & $78 \pm 8$ & $386 \pm 29$ \\
& $80 \pm 8^{\text {a }}$ & $336 \pm 126^{\mathrm{a}}$ & $595 \pm 109^{\mathrm{a}}$ \\
\hline
\end{tabular}

Groups of 5 rats received vehicle or tyrosine $(200 \mathrm{mg} / \mathrm{kg}$, ip), and were killed 1-hour later. Animals had been pretreated with either nothing (No Reserpine) or reserpine (Chronic Reserpine), $2.5 \mathrm{mg}$ / $\mathrm{kg}$ twice daily for 4 days; on the morning of the fifth day, the last injection of reserpine was given 2 hours before tyrosine administration. All injections were intraperitoneal. Data are presented as the means \pm SEM

a $\mathrm{P}<0.05$ compared to vehicle values in the Chronic Reserpine group (adapted from [28])

prolactin release-inhibiting factor. (For example, drugs that enhance dopaminergic transmission [like L-dopa, which like tyrosine, is converted to DA] suppress prolactin secretion; drugs that block DA transmission enhance release of the hormone [32].)
Tyrosine has also been employed with some success to ameliorate depression [33]. This usage is based on the premise that some forms of this disease may be caused by diminished functioning of noradrenergic neurons, and thus be treatable by agents that increase transmission at $\mathrm{NE}$ synapses. The amino acid has also been applied with limited success to the treatment of Parkinson's disease, with the hope that it would act similarly to L-dopa by increasing DA synthesis and release in the corpus striatum [34].

It is thus possible that tyrosine may ultimately be used in the treatment of a number of diseases involving reduced transmission at catecholaminergic synapses. As a naturally-occurring compound (and a normal part of the diet), its use seems more likely to be associated with fewer side effects than occurs with the use of drugs.

\section{Acetylcholine}

Acetylcholine is synthesized from choline in a singlestep reaction catalyzed by choline acetyltransferase (CAT) (Fig. 1, bottom panel). This enzyme shares with the aromatic amino acid hydroxylases the property that, at normal brain substrate levels, the enzyme is not fully saturated. Hence, increases in brain choline levels readily stimulate brain acetylcholine formation (Fig. 3) [35]. (This relationship not only holds for brain neurons, but also for autonomic nerves. For example, the administration of choline increases acetylcholine [ACh] levels in preganglionic neurons innervating the adrenal medulla [36].) Choline gains access to the brain by a transport carrier that, to date, does not appear to be shared with other, naturally-occurring compounds. Hence, the blood choline level seems to be the important determinant of brain choline uptake (see [37]).

Choline levels in blood derive principally from two sources - choline synthesized in the liver, and choline cleaved from dietary lecithin (phosphatidylcholine) in the course of digestion and absorption of fats. The dietary source of choline is quite important (about $40-50 \%$ of the choline added to the body each day derives from the diet, the rest coming from liver synthesis [38]), and consequently, it was not surprising to find that blood choline, brain choline, and brain acetylcholine levels all rise following the ingestion of a lecithin (phosphatidylcholine)-containing meal [39].

Choline- or lecithin-induced increases in neuronal acetylcholine levels lead to enhanced release of transmitter, when cholinergic neurons depolarize. Using the isolated, phrenic nerve-diaphragm preparation, Bierkamper and Goldberg [40] 


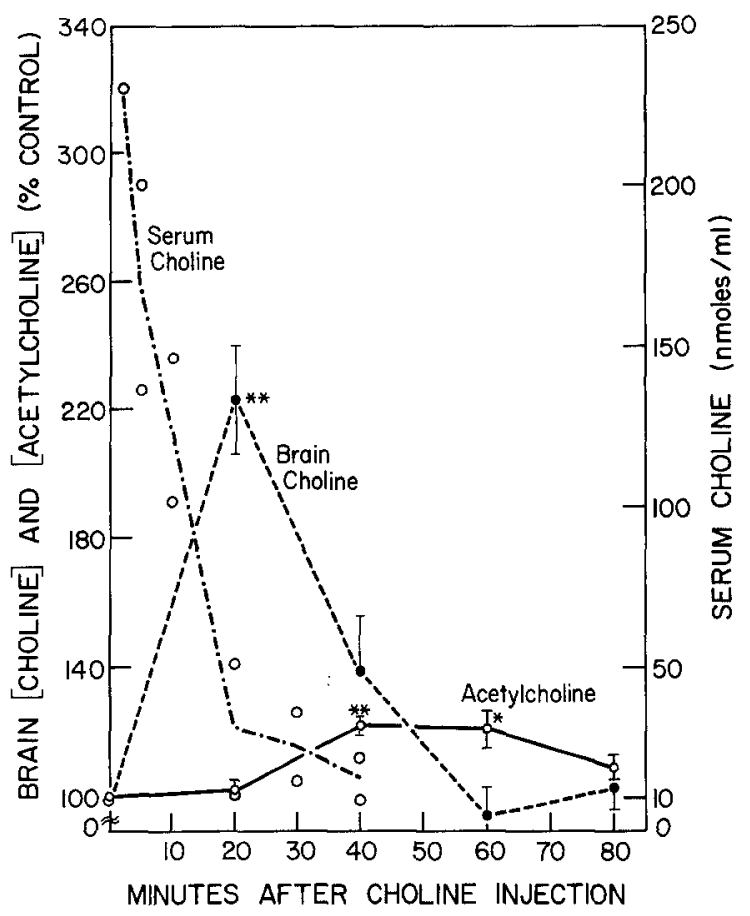

Fig. 3. Time-course of the response of serum choline, brain choline, and brain acetylcholine to choline administered by injection. Groups of 5-9 male rats received choline chloride $(60 \mathrm{mg} / \mathrm{kg}$, ip) or diluent. At various intervals thereafter, they were killed by microwave irradiation of the head, and whole brain choline and acetylcholine levels determined. Blood choline levels were measured in rats treated identically, but killed by decapitation. Bars indicate standard errors; open circles indicate the range of values for serum choline. $* \mathbf{P}<0.01$, and $* * \mathbf{P} 0.001$ compared to values in rats injected with diluent. (From [43])

found the amount of acetylcholine released into the medium following electrical stimulation to be doserelated to the choline content of the medium; and, the release of epinephrine and norepinephrine by the adrenal medulla in animals subjected to insulininduced hypoglycemia, cold, or other stresses has been found to be enhanced in animals pretreated with choline [41].

At present, the underlying physiologic reasons for the tying of acetylcholine synthesis by neurons to choline (lecithin) availability in the diet is not understood. Notwithstanding, this has not stalled the application of choline and lecithin as therapeutic agents for the treatment of disease states thought to involve hypofunctioning of cholinergic neurons. The most impressive results have been obtained with the treatment of tardive dyskinesia, a neurological disorder that often responds to the administration of physostigmine, an inhibitor of acetylcholinesterase (see [37]). Other neurological diseases have been treated with choline or lecithin, with less impressive results to date; these include Huntington's disease, Friedrich's ataxia, and Parkinson's disease. One promising use for choline/lecithin may be in the treatment of memory disorders, which have been reported to respond to physostigmine (see [37]). Some preliminary results suggest this to be the case.

Acknowledgements. Some of the studies described were supported in part by grants from the NIH, NIMH, NASA, the American Diabetes Association, and the Ford Foundation. Dr. Fernstrom is the recipient of a Research Scientist Development Award from the NIMH (MH00254).

\section{References}

1. Cooper JR, Bloom FE, Roth RH (1978) The Biochemical Basis of Neuropharmacology, third ed, Chapter 8. Oxford University Press, New York

2. Kaufman S (1974) Properties of pterin-dependent aromatic amino acid hydroxylases. In: Wolstenholme, GEW, FitzSimons DW, Aromatic amino acids in the brain. Associated Scientific Publishers, Amsterdam, 85-108

3. Fernstrom JD, Wurtman RJ (1971) Brain serotonin content: physiological dependence on plasma tryptophan levels. Science 173: 149-152

4. Fernstrom JD, Faller DV, Shabshelowitz H (1975) Acute reduction of brain serotonin and 5HIAA following food consumption: correlation with the ratio of serum tryptophan to the sum of competing neutral amino acids. J Neural Transm 36: 113-121

5. Pardridge WM (1977) Regulation of amino acid availability to the brain. In: Wurtman RJ, Wurtman JJ (eds) Nutrition and the brain, vol. I. Raven Press, New Yorks, p 141-204

6. Fernstrom JD, Wurtman RJ (1971) Brain serotonin content: increase following ingestion of carbohydrate diet. Science 174 : 1023-1025

7. Fernstrom JD, Wurtman RJ (1972) Brain serotonin content: Physiological regulation by plasma neutral amino acids. Science 178: 414-416

8. Fernstrom JD (1977) Effects of the diet on brain neurotransmitters. Metabolism 26: 207-223

9. Madras BK, Cohen EL, Messing RB, Munro HN, Wurtman RJ (1974) Relevance of free trytophan in serum to tissue tryptophan concentrations. Metabolism 23: 1107-1116

10. Curzon G, Knott PJ (1974) Effects on plasma and brain tryptophan in the rat of drugs and hormones that influence the concentration of unesterified fatty acids in plasma. Br J Pharmacol 50: 197-204

11. Pardridge WM (1979) The role of blood-brain barrier transport of tryptophan and other neutral amino acids in the regulation of substrate-limited pathways of brain amino acid metabolism. J Neural Transm [Suppl] 15: 43-54

12. Wahren J, Felig P, Hagenfeldt L (1976) Effect of protein ingestion on splanchnic and leg metabolism in normal man and in patients with diabetes mellitus. J Clin Invest 57: 987-999

13. MacKenzie RG, Trulson ME (1978) Effects of insulin and streptozotocin-induced diabetes on brain tryptophan and 5HT metabolism in rats. J Neurochem 30: 205-211

14. Curzon G, Fernando JCR (1977) Drugs altering insulin secretion. Effects on plasma and brain concentrations of aromatic amino acids and on brain 5-hydroxytryptamine turnover. $\mathrm{Br} \mathrm{J}$ Pharmacol 60: 401-408 
15. Crandall EA, Fernstrom JD (1980) Acute changes in brain tryptophan and serotonin after carbohydrate or protein ingestion by diabetic rats. Diabetes 29: 460-466

16. Wurtman JJ, Wurtman RJ (1979) Drugs that enhance serotonergic transmission diminish elective carbohydrate consumption by rats. Life Sci 24: 895-904

17. Ashley DVM, Anderson GH (1975) Correlation between the plasma tryptophan to neutral amino acid ratio and protein intake in the self-selecting weanling rat. $\mathbf{J}$ Nutr 105: $1412-1421$

18. Hartmann E (1977) L-tryptophan: a rational hypnotic with clinical potential. Am J Psychiatr 134: 366-370

19. Sved AF, Fernstrom ID (1980) Tryptophan administration lowers blood pressure in spontaneously hypertensive rats. Fed Proc 39: 608 (abstract)

20. Arnold MA, Fernstrom JD (in press) L-tryptophan injection enhances pulsatile growth hormone secretion in the rat. Endocrinology

21. Woolf PD, Lee L (1977) Effect of the serotonin precursor, tryptophan, on pituitary hormone secretion. J Clin Endocrinol Metab 45: 123-133

22. Arnold MA, Fernstrom JD (1978) Serotonin receptor antagonists block a natural, short-term surge in serum growth hormone levels. Endocrinology 103: 1159-1163

23. Arnold MA, Fernstrom JD (1979) Tryptophan injection stimulates growth hormone secretion in rats. Proc. 61st Meeting of the Endocrine Society (US), p 223

24. Martin JB, Durand D, Gurd W, Faille G, Audet J, Brazeau P (1978) Neuropharmacologic regulation of episodic growth hormone secretion in the rat Endocrinology 102: 106-113

25. Cooper JR, Bloom FE, Roth RH (1978) The biochemical basis of neuropharmacology, third ed, Chapters 6 and 7. Oxford University Press, New York

26. Wurtman RJ, Larin F, Mostafapour S, Fernstrom JD (1974) Brain catechol synthesis: control by brain tyrosine concentration. Science 185: 183-184

27. Sved AF, Fernstrom JD, Wurtman RJ (1979) Tyrosine administration reduces blood pressure and enhances brain norepinephrine release in spontaneously-hypertensive rats. Proc Natl Acad Sci USA 76: 3511-3514

28. Sved AF, Fernstrom JD, Wurtman RJ (1979) Tyrosine administration decreases serum prolactin levels in chronicallyreserpinized rats. Life Sci 25: 1293-1300

29. Gibson CJ, Wurtman RJ (1978) Physiological control of brain norepinephrine synthesis by brain tyrosine concentration. Life Sci 22: 1399-1406

30. Fernstrom JD, Faller DV (1978) Neutral amino acids in the brain: changes in response to food ingestion $\mathrm{J}$ Neurochem 30 : $1531-1538$

31. Davies DS, Reid JL (1975) Central Action of drugs in blood pressure regulation. University Park Press, Baltimore, p 306
32. Fernstrom ID, Wurtman RJ (1977) Brain monoamines and reproductive function. In: Greep RO, (ed) Reproductive physiology II. University Park Press, Baltimore p 23-56

33. Gelenberg A, Wojcik JD, Growdon JH, Sved AF, Wurtman RJ (1980) Tyrosine for the treatment of depression. Am J Psychiatr 137: 622-623

34. Growdon $\mathrm{JH}$, Melamed $\mathrm{E}$ (in press) Biochemical effects of tyrosine administration to patients with Parkinson's disease. Neurology (Minneap)

35. Cohen EL, Wurtman RJ (1975) Brain acetylcholine: increase after systemic choline administration. Life Sci 16: 1095-1102

36. Ulus IH, Hirsch MJ, Wurtman RJ (1977) Trans-synaptic induction of adrenomedullary tyrosine hydroxylase activity by choline: evidence that choline administration increases cholinergic transmission. Proc Natl Acad Sci USA 74: 798-800

37. Pardridge WM, Cornford EM, Braun LD, Oldendorf WH (1979) Transport of choline and choline analogues through the blood-brain barrier. In: Barbeau A, Growdon $\mathrm{JH}$, Wurtman RJ (eds) Nutrition and the brain, volume V. Raven Press, New York, p 25-34

38. Zeisel S, Bluztajn JK, Wurtman RJ (1979) Brain lecithin biosynthesis: evidence that bovine brain can make choline molecules. In: Barbeau A, Growdon JH, Wurtman RJ (eds) Nutrition and the brain, volume V. Raven Press, New York, p 47-56

39. Hirsch MJ, Wurtman RJ (1978) Lecithin consumption increases acetylcholine concentrations in rat brain and adrenal gland. Science 202: 223-224

40. Bierkamper GG, Goldberg AM (1979) Effect of choline on the release of acetylcholine from the neuromuscular junction. In: Barbeau A, Growdon JH, Wurtman RJ (eds) Nutrition and the brain, volume V. Raven Press, New York, p 243-252

41. Scally MC, Ulus IH, Wurtman RJ (1978) Choline administration to the rat increases urinary catecholamines. J Neural Transm 43: 103-112

42. Arnold MA (1979) Neural regulation of growth hormone secretion in the male rat. PhD Thesis, Massachusetts Institute of Technology

43. Cohen EL (1976) In vivo studies of the effect of the availability of choline on the biosynthesis and content of acetylcholine in brain. PhD Thesis, Massachusetts Institute of Technology

John D. Fernstrom, Ph. D.

Associate Professor of Physiology

Department of Nutrition and Food Scunce

Massachusetts Institute of Technology

Cambridge, MA 02139

USA 


\section{Discussion after Fernstrom's Presentation}

Goldman: In the experiment you described where hypertensive rats were given tyrosine and their blood pressure was lowered, why didn't the rest of the brain have altered levels of NE and its metabolites? Wouldn't the TYR have access to all catecholaminergic neurons of the brain instead of only the blood pressure controlling ones in the brain stem?

Fernstrom: It did get into all of the neurons. Catecholaminergic neurons are more precursor dependent the more active they are. If drugs like $\alpha$ methyl dopa and clonidine act at selectively the brain stem to stimulate NE receptors (although they have free access to all NE receptors) and somehow lower blood pressure, then these noradrenergic neurons of the brain stem could ultimately be influencing the activity of on other, descending noradrenergic neurons. It's pretty well known that these descending noradrenergic neurons stimulate sympathetic outflow and can cause increased blood pressure. If these other hypothetical brainstem neurons which are noradrenergic function to lower blood pressure, they might be extremely active in the hypertensive animal in attempting to reduce the blood pressure. If you now provide them with excess tyrosine, they may make considerably more $\mathrm{NE}$ and thereby cause a greater reduction of activity in the descending noradrenergic neurons and ultimately cause a reduction of blood pressure. Therefore, in this particular model, the brainstem catecholaminergic neurons which act to lower blood pressure are more prepared to synthesize and retense more NE in response to an increase of available tyrosine.

It's even easier to see the dependency of a tyrosine effect upon activity in dopaminergic neurons. Dopaminergic neurons which are not particularly active are not precursor dependent. That's why we treated animals with reserpine. It depletes the stores of DA and thereby stimulates new synthesis of DA. The neurons become maximally precursor dependent and our other manipulations with tyrosine to raise $\mathrm{DA}$ become effective.

Powley: According to what you say, tyrosine administration should have no effect in normotensive animals.

Fernstrom: It does have have an effect, but it is very small in comparison. Normotensive rats have around $110 \mathrm{~mm}$ of Hg. A $200 \mathrm{mg} / \mathrm{kg}$ dose of tyrosine will lower it by only around $10 \mathrm{~mm}$ of $\mathrm{Hg}$, and our method of obtaining pressure estimates, via a tail cuff, is only accurate to 5 or $10 \mathrm{~mm}$ of $\mathrm{Hg}$.

Nicolaidis: As you know, it is difficult to increase sleeping time of animals. We have recently shown that when we administer glucose plus insulin to animals, they sleep more. If the animals have been previously deprived, there is an even larger increase of sleep time, up to $47 \%$ over normal. This is therefore consistent with your hypothesis since this manipulation should have caused increased brain 5HT and it is associated with increased sleep.
Fernstrom: Your results are very interesting. For years, we've tried to get sleep researchers here in the U.S. interested in studying metabolic effects like you describe, but so far such projects have had low priority. Perhaps your studies will stimulate more research here.

Novin: There are large quantities of 5HT in the gastrointestinal system. Are there parallel changes in gut 5HT as in the brain? Does gut 5HT change with diet, or are there other functional changes in that pool?

Fernstrom: Dick Wurtman had a graduate student who worked on that problem and found that urinary 5-HIAA could be increased by tryptophan administration. Since most urinary 5-HIAA is gut derived, this suggests that gut 5HT synthesis might be sensitive to changes in precursor level. Urinary 5-HIAA level also appeared to vary directly with the protein content of a meal (Metabolism (1979) 28: 820; J Neurochem (1977) 29: 267).

Pardridge: Anderson (J. Nutrition (1975) 105: 14121421) showed an inverse relationship between protein drive and plasma tryptophan levels. Can you comment upon this?

Fernstrom: Yes, his observations are rather interesting. The Wurtmans are also working on appetite and 5HT, but from a different point of view. The two groups have reached different conclusions, so it's worth pointing out both of them here. Anderson gave weanling rats access to a selection of proteins. The idea is that selection is much more natural than the present laboratory practice of providing all the animal's nutritional needs in a small block of food. Such experiments can show only how much the animal eats. The selection experiment allows a finer degree of regulation to be manifest. Anderson used a number of different proteins, some of which were not deficient in any amino acids, like casein, and some of which were deficient in specific amino acids, like gluten. He added some amino acids to some and not to others so that there was considerable variability of specific ratios among the various amino acids consumed. A given rat had a choice between two such diets which contained different contents of the same protein. He found an inverse relationship between the amount of protein selected in the diet (actually the ratio of protein to total dietary calories) and the tryptophan to LNAA ratio; i. e., the higher the ratio, the less protein was selected in the diet. He has found a comparable relationship when comparing the intakes of various subgroups of humans. Anderson, has also examined the food intakes of Canadians over the past 80 years. Canada keeps detailed records of what is purchased in the stores, and from these, Anderson calculated that the average per capita protein intake has remained remarkably constant over the years. He has postulated that lean body mass is very tightly regulated and that variations of protein intake may be the means for keeping it constant. He apparently worked out the Canadian protein consumption data first, and the relationship led him to do the studies where he varied the diets of the 
rats. He's also looked at other models, such as diabetic rats, and all show predictable results.

The Wurtmans (Science (1977) 198: 1178) do their experiments very differently. The rats are deprived of food for 16 hours each day. Food is available only for the 8 hours of darkness. After weight stabilizes, they give the rats a choice between two diets with varying protein or carbohydrate content. The main focus of the experiments is to ascertain the effects of drugs on selection. When they administer amphetamine, a drug with mainly catecholaminergic effects, it causes a decrease of chow intake over the 8 hours, and when the rats have a choice, it reduces protein and carbohydrate intake comparably. In other words, the selection data were comparable to the intake of chow alone. Fenfluramine is another anorectic agent, but it is thought to effect serotonergic neurons more than catecholaminergic neurons. Like amphetamine, it caused a reduction of chow intake, but it also caused a selective decrease of carbohydrate intake. Over the 8-hour feeding period, the animal kept its protein intake constant but reduced its carbohydrate intake by one half to two thirds. So in the Wurtmans' experiments, it seems to be carbohydrate which is variable or expendable in the diet, not protein. Anderson, on the other hand, claims that dietary protein is what is actually regulated. It all seems to depend on how the experiments are done. From the Wurtmans' data, one can hypothesize a model regulatory loop where an animal consumes excess carbohydrate, its plasma amino acids shift to favor brain tryptophan uptake, and more 5HT is made in the brain. The result is analogous to what happens when a 5 HT agonist is given. The animal eats less carbohy- drate. So there are two possible regulatory feedback loops hypothesized here. Which is more important is not presently known.

Havrankova: How does insulin increase blood tryptophan?

Fernstrom: It's not really known. One guess is that the insulin somehow acts at the muscle to cause a selective release of tryptophan. The rat has $5 \mathrm{ml}$ of blood for each $100 \mathrm{~g}$ of weight, so if blood tryptophan is normally $20 \mu \mathrm{g} /$ $\mathrm{ml}$, a $200 \mathrm{~g}$ rat has $200 \mu \mathrm{g}$ of tryptophan in the blood. When insulin goes up, that can double. Because the muscle has such a large pool of available amino acids, such a change would be hard to detect. We tried to do the experiments using various labelled compounds, but we were never able to find much given the noise in the muscle amino acid pods.

Oomura: What happens to brain $5 \mathrm{HT}$ when you administer insulin but do not let the animal eat?

Fernstrom: It goes up. We actually did those experiments before we did the feeding studies (Metabolism (1972) 21: 337).

Pardridge: Have you tried varying other sugars, like fructose?

Fernstrom: Yes, we've given several. Most didn't do much, including sorbitol and mannose. Fructose actually increased brain $5 \mathrm{HT}$, but I suspect that most of it was converted to glucose in the liver. In general, those that led to an increase of plasma glucose, like sucrose, induced insulin secretion and led to an increase of brain 5HT. 